\title{
La aplicación del color sobre la arquitectura moderna: Revisión de los proyectos de Bruno Taut y Le Corbusier
}

\author{
The application of colour to modern architecture: A \\ review of projects by Bruno Taut and Le Corbusier
}

\author{
Resumen
}

Autoras:

Aurora Alcaide-Ramírez* alcaide@um.es

Ana Ruiz-Abellón* anaruizabellon@gmail.com

*Universidad de Murcia

Recibido: 30/Sep/2020 Aceptado: 14/Dic/2020
- a arquitectura y la pintura se encuentran en un momento de cambio en la etapa inicial del siglo XX. La llegada de la Modernidad o Estilo Internacional supuso un replanteamiento en las formas de construir, pues materiales como el hormigón, el hierro o el vidrio aparecieron en escena condicionando, además del proceso constructivo, la estética de la edificación. Pero no solo ello caracteriza dicho cambio. El color, tradicionalmente asociado al ámbito pictórico, pasa a formar parte de la arquitectura como un recubrimiento, aplicándose acorde con el fin que persigue el diseñador, que en este caso es también el arquitecto. Para poner en contexto este cambio estético se van a analizar dos proyectos, uno de Bruno Taut y otro de Le Corbusier.

Palabras clave: arquitectura moderna; pintura; color; Le Corbusier; Bruno Taut

\section{Abstract:}

Architecture and painting are at a turning point in the early part of the 20th century. The arrival of Modernity or International Style meant a rethinking of the ways of building, since materials such as concrete, iron or glass appeared on the scene, conditioning not only the constructive process, but also the aesthetics of the building. But this is not the only characteristic of this change. Colour, traditionally associated with the pictorial field, became part of architecture as a covering, which is applied in accordance with the purpose pursued by the designer, who in this case is also the architect. To put this aesthetic change into context, two projects will be analyzed, one by Bruno Taut and other by Le Corbusier.

Keywords: modern architecture; painting; colour; Le Corbusier; Bruno Taut. 


\section{Introducción}

En el presente artículo se aborda la cuestión de cómo el color pasa a ser un elemento pictórico destacable en el ámbito arquitectónico en las primeras décadas del siglo $\mathrm{XX}$, centrando la atención en la arquitectura moderna (también denominada Estilo Internacional o Funcionalismo), desarrollada desde mediados de los años veinte hasta finales de los años sesenta. Un movimiento que supuso un cambio en la arquitectura de magnitud comparable con la del Renacimiento italiano, según apunta Jorge Sainz (1997). Para este autor, las transformaciones que se produjeron se hicieron presentes en tres aspectos de la arquitectura que se corresponden con tres categorías vitruvianas: firmitas, utilitas y venustas, traducidas como la técnica constructiva, el contenido funcional y la composición formal. Asimismo, el vidrio, el acero y la técnica del hormigón armado se incorporaron al lenguaje arquitectónico (Sainz, 1997). Además, se busca en este tipo de arquitecturas la regeneración de la sociedad a través de esta renovación arquitectónica, por ejemplo, con los proyectos destinados a barrios obreros (como es el caso de los dos ejemplos propuestos en el apartado de Casos de estudio). $Y$ en lo relativo al cambio formal se elimina todo elemento ornamental y los volúmenes se presentan nítidos, sin elementos de distracción, predominando las geometrías simples: "la recta como fundamento y la curva como contrapunto" (Sainz, 1997, p.265)

La arquitectura del primer cuarto del siglo XX siente la necesidad de adaptarse a las tendencias del momento e impulsa cambios, tal y como se ha comentado anteriormente, así como también se ve condicionada por el contexto, sobre todo por sucesos como la pandemia de tuberculosis sufrida a principios de los años veinte (Torrico, 2020). En virtud de esta, el diseño arquitectónico se adapta a unas condiciones de vida diferentes, en las que se necesita la amplitud de los espacios, así como la estancia al aire libre. Los grandes ventanales de cristal pasan a ser característicos en esta etapa de cambio, permitiendo la entrada de luz y la correcta ventilación de las habitaciones. Se eliminan las separaciones interiores innecesarias, dotando de una mayor amplitud espacial a las estancias interiores, e inclusive los muebles se diseñan acordes a esta situación, eliminando sus detalles ornamentales para facilitar su limpieza y potenciar su ergonomía. El uso del color blanco en las paredes interiores y exteriores de los edificios también se extiende en esta época debido a sus propiedades higiénicas.

El empleo de determinados materiales como el hierro, el vidrio, el cemento o la madera permite crear formas geométricas simples, así como la entrada de la luz exterior al interior de los habitáculos (mediante ventanas, claraboyas, etc.), además de aligerar el peso de la edificación sin renunciar a la resistencia. Por otra parte, su textura y tonalidad propia condiciona, en muchos casos, el acabado final de los edificios en los que se incluyen. Esta revolución material, que afecta a la disciplina arquitectónica, tiene lugar en un momento donde la pintura también se ve involucrada en un proceso de cambio en su metodología (Mehaffy y Salingaros, 2020). El Movimiento moderno rompe con los patrones de edificación y precedentes tradicionales, especialmente en Europa y América, gracias a los arquitectos Walter Gropius, Ludwig Mies van der Rohe, Le Corbusier, Bruno Taut, Jacobus Johannes Pieter Oud, Richard Neutra, Rudolf Schindler, Philip Johnson, Alvar Aalto, Eliel y Eero Saarinen, Erik Gunnar Asplund, Josep Lluís Sert, Louis Kahn, Pier Luigi Nervi, Gio Ponti, Kenzō Tange, Lúcio Costa y Oscar Niemeyer.

Otra característica relevante del Movimiento moderno es la supresión de todo elemento en el revestimiento de las arquitecturas, lo que conlleva la imposibilidad de apreciar alguna marca que aluda a la pertenencia geográfica o cultural de la construcción, a favor de elementos plásticos como la masa, superficie, tiempo, espacio, luz, color, material, etc. En la arquitectura del Movimiento moderno se impone, por tanto, la funcionalidad y la libertad estética o informidad (Mújica, 2014). En este sentido, cabe puntualizar que la forma sigue a la función: esta determina la forma de las construcciones.

En la mayoría de los edificios contextualizados dentro de esta tendencia, prima el uso del blanco en el revestimiento exterior o de la tonalidad de los materiales constructivos. No son muy comunes los casos en los que el color cobra un papel protagonista en el diseño compositivo. Cuando ocurre esto, se distinguen dos formas de proceder: aplicar el color con materia pictórica sobre la superficie arquitectónica a modo de añadido, frente a la exaltación del color natural de los materiales constructivos: hormigón, hierro, madera y vidrio, imponiéndose finalmente esta última (Premier y Gasparini, 2017).El objetivo principal de este artículo es analizar algunos ejemplos contextualizados dentro de la primera posibilidad, para identificar sus principales características, qué criterios se siguen a la hora de aplicar un determinado color $u$ otro, de qué manera se produce esta aplicación y con qué finalidad, qué relaciones se establecen entre el cromatismo exterior del edificio, el del entorno o el dispuesto en su interior, cómo afecta a la percepción de los volúmenes de una construcción la presencia de colores en sus paramentos exteriores, o cómo se articula el color con la tonalidad y textura de los materiales constructivos y con los paños totalmente blancos, entre otros aspectos significativos.

\subsection{Antecedentes cromáticos del Movimiento Moderno}

En el estudio de la unión pintura-arquitectura, destaca un movimiento pictórico en el que el color y la abstracción formal son los elementos clave: el Neoplasticismo. El proceso de abstracción del color que se produce en este movimiento afecta a la estética de la arquitectura, constituyendo un referente esencial para poder valorar y analizar el empleo del color en la arquitectura moderna; pero, además, muchos de los principios en los que se 
fundamenta también son comunes a esta última, de ahí que se dedique un apartado a su estudio.

La aplicación del color en la superficie arquitectónica (ya sea pared, suelo o techo) lleva consigo el planteamiento de unas estrategias que afectan a la toma de decisiones, tanto de arquitectos como de artistas (en el caso de colaborar en un proyecto). Estas decisiones afectan además a la estética resultante de la arquitectura, así como a su composición, pues en el caso de que el diseño cromático se realice durante la etapa del boceto, puede implicar cambios en la distribución del espacio. Este planteamiento, entre otros, propicia la ruptura con la arquitectura clásica en la segunda década del siglo XX. Para Villalobos (2002), la sensibilidad visual por la aplicación del color en la arquitectura no había podido alcanzar todo su esplendor antes del siglo XX, quedando mermada por el empleo exacerbado de los elementos ornamentales que recubrían las fachadas.

Los cambios estéticos que se produjeron en la arquitectura del Movimiento Moderno, fueron impulsados inicialmente por la arquitectura Neoplasticista, en la que se prolongan los principios pictóricos de dicho movimiento (Mondrian, 1983). Theo van Doesburg, uno de los máximos representantes de Neoplasticismo pictórico y arquitectónico, establece 17 características de la arquitectura de este movimiento que son presentadas en el número 12 de la revista De Stijl, de 1924, y posteriormente en el número de otoño/invierno de la revista L'Architecture Vivante, de 1925. Entre ellas destacan, por ser compartidas por la arquitectura moderna:

2. Partir de los elementos: masa, luz, materiales, planos, tiempo, espacio, color, etc.

8. Eliminar la dualidad interior-exterior.

9. Abrir la arquitectura al espacio público.

13. Reemplazar la simetría por el equilibrio de partes desiguales. 14. Desarrollar plásticamente un poliedro en el espacio-tiempo. 15. Eliminar las obras ilusionistas a favor de los planos de color. 16. Luchar contra cualquier indicio de aspecto decorativo.

17. Hacer de la arquitectura una síntesis de la construcción plástica. (Van Doesburg, citado en Diecisiete puntos de la arquitectura neoplasticista, 2012, párrs. 1-17).

Lo destacable del movimiento neoplasticista es la conjugación que se establece entre arquitectura y pintura, rasgo también presente en los proyectos de Bruno Taut y Le Corbusier seleccionados como casos de estudio. El espacio arquitectónico se abre hacia el exterior y los interiores se vuelven diáfanos, empleando únicamente las paredes necesarias para la división mínima de las estancias. Las formas se simplifican, potenciando su geometría y aboliendo los elementos ornamentales comunes en el revestimiento de las arquitecturas de estilo clásico (Tournikiotis, 2001). Este proceso de abstracción, simplificando las formas y potenciando el color, se aprecia muy claramente en las contraconstrucciones o diseños que realizaban los artistas neoplasticistas en sus colaboraciones con los arquitectos del momento (Figura 1). Estos diseños cromáticos servían de estudio previo para la toma de decisiones acerca de la distribución de los planos de color (Tournikiotis, 2001), siguiendo un patrón: la profundidad se relacionaba con el color azul, la altura con el rojo y anchura con el amarillo (Mújica, 2014).

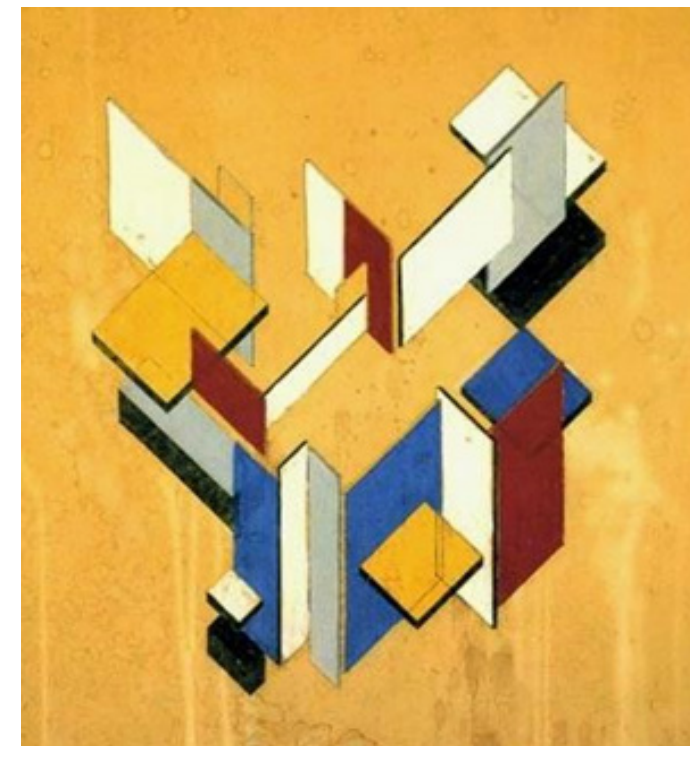

Figura 1: Theo van Doesburg y Cornelius van Eesteren, Contra-construcción de la Maison Particuliere, 1923. Dibujo axonométrico a color de la cara donde se encuentra la habitación de invitados

Fuente: http://unalhistoria3.blogspot.com/2014/11/ casa-particular-maison-particuliere.html

El resultado plástico de las colaboraciones entre arquitectos y artistas fue bastante rico. El problema surgió a la hora de tomar decisiones conjuntas acerca de los diseños propuestos. La jerarquía que imponían los arquitectos en cuanto a los resultados que eran más adecuados para el proyecto mermaba las ideas de los artistas, a quienes el arquitecto atribuía diseños demasiados cargados de color o con una distribución de los planos cromáticos que podía llevar a pensar en la ausencia de un estudio previo (Serra, 2010). Sin embargo, en opinión de Piet Mondrian, "la arquitectura sirve a la pintura, hasta el punto de que podemos hablar del modelado de un lienzo tridimensional, más que de arquitectura" (1983, p. 79). Esta idea de lienzo tridimensional es la que explotan los artistas neoplasticistas a la hora de afrontar los proyectos arquitectónicos, ya sea en solitario o en colaboración con los arquitectos.

La apertura del cubo que propone la arquitectura neoplasticista y moderna permite eliminar características como la simetría y la repetición a favor de una relación equilibrada de factores, como la dimensión, la proporción y la situación, de modo que en el diseño final una pared no es más importante que la otra, tal y como ocurría con la fachada en la arquitectura clásica, cuyo grado de importancia era mayor que la del resto de las paredes exteriores. Por otra parte, la inclusión del color sobre la superficie arquitectónica, tanto interior como exterior, se plantea teniendo en consideración estos factores de espacio y tiempo, puesto que el color es un medio orgánico de expresión propio de la arquitectura (Mújica, 
2014). Estos principios afectan directamente a la toma de decisiones respecto a la distribución de los planos de color, así como a la forma de los mismos, dependiendo incluso de la función que desempeñe la construcción, la cual se puede encontrar tanto en fase de boceto como en la obra terminada.

\section{Método}

Para llevar a cabo la investigación, que en este caso es multidisciplinar (Munárriz, 2013) porque aúna dos disciplinas distintas, pintura y arquitectura, se plantea una serie de cuestiones relacionadas con la investigación de tipo cualitativo (Hernández, Fernández y Baptista, 2010), que son: ¿cómo se ha aplicado el color sobre la arquitectura moderna? ¿influye el estadio de la construcción para el desarrollo del diseño cromático? ¿es perceptible en el resultado si el diseño se ha llevado a cabo por arquitectos o por artistas, así como por la colaboración de ambos?

Para abordarlas, la metodología que se plantea es contextualizar la arquitectura moderna y tratar aspectos de ella como la aplicación del color en su revestimiento, en este caso, con el apoyo de dos proyectos arquitectónicos en donde el diseño cromático se ha realizado por los propios arquitectos: el Gartenstadt Falkenberg, realizado por Bruno Taut entre 1920 y 1930, y los Quartiers Modernes Frugès (1924-1926), obra de Le Corbusier. El primero de ellos se selecciona como caso de estudio por su excepcionalidad, ya que se trata de un proyecto cuyo volumen abarca alrededor de 1500 viviendas y una estimación de 7500 residentes. En cuanto al color, se articula en las edificaciones que integran este conjunto residencial conforme a planos geométricos que no se limitan al formato rectangular de los paramentos, sino que en algunos casos adquieren formas triangulares, romboidales o cuadrangulares, modulando la fachada principal de las construcciones, en diálogo con otros elementos, como vanos o molduras. La paleta cromática utilizada en este proyecto es, asimismo, bastante amplia (teniendo el color blanco una presencia mínima), lo que hace destacar a esta urbanización berlinesa, nombrada Patrimonio Cultural de la Humanidad en 2008, entre otras de su entorno.

En cuanto al proyecto de Le Corbusier, se ha elegido como objeto de estudio, además de por su particular inclusión del color (elemento no muy común en su prolífica trayectoria arquitectónica), debido a su función residencial y a su concepción como ciudad jardín, al igual que las viviendas seleccionadas de Taut, lo que favorece el paragone entre ambas construcciones. Le Corbusier es, además, una figura paradigmática dentro del Estilo Internacional, siendo imprescindible en cualquier análisis que se lleve a cabo sobre este movimiento. Este arquitecto "purifica la arquitectura", la dota de rigor y austeridad, aportándole su propia impronta, según señala Daniel Villalobos (2002), características que se identifican en las casas que integran Quartiers Modernes Frugès. Por otra parte, destacamos su faceta de pintor que, aunque menos conocida, desarrolla en paralelo a la de arquitecto, y que le aporta una particular mirada, que se percibe en cómo articula el cromatismo de los paramentos exteriores de las casas unifamiliares de conjunto residencial objeto de estudio, en donde el color blanco (que alude a la pureza y austeridad comentada anteriormente) convive con tonalidades poco saturadas.

Para el análisis de los dos proyectos se tienen en cuenta los factores o estrategias de color definidos por Juan Serra (2010) y Malvina Arrarte (2008). Según la clasificación establecida por el primero, las estrategias de color pueden establecerse en tres grupos: en el primero, el color interfiere en la percepción de las propiedades visuales de la forma, el segundo describe el objeto arquitectónico y el tercero trata del aspecto decorativo del color (el color por su valor intrínseco). Las propiedades visuales de la forma, textura aparente, peso visual, dimensiones, distorsión geométrica, integración o desintegración de los componentes de la forma arquitectónica, mímesis entre la arquitectura y el entorno, y dependencia de factores como la iluminación o el contexto histórico, están relacionadas con el primer grupo, mientras que la forma y función derivan del segundo. En el caso del tercer grupo, tal y como apunta el autor, hablar de "color decorativo" en la arquitectura contemporánea puede ser confuso porque parece no encajar con los materiales aplicados en dicha etapa. En la arquitectura moderna e color se aplica como una capa añadida a la superficie, la cual está totalmente desprovista de ornamentación, y además permite la no conexión tanto con el soporte como con el entorno, puesto que "el color decorativo es aquel que es autojustificativo, que prescinde de explicaciones" (Serra, 2010, p. 89).

Por otra parte, Malvina Arrarte (2008) plantea la viabilidad de distintos tipos de coloración: plana, volumétrica, capa, estructural o sintáctica, decorativa, neutralizadora y blanca. Esta clasificación se basa en la relación con el espacio/soporte que los contiene, de manera que se produce una unión entre ambos; por ejemplo, si el color parece artificial en el espacio donde se ubica, este no estaría en conexión con la superficie que lo contiene, según la autora. Desde nuestro punto de vista, los tipos de coloración que propone Arrarte complementan a las estrategias cromáticas de Serra vistas anteriormente. El color puede reducir el objeto a los elementos planos de los que se compone, reforzar su tridimensionalidad, ser aplicado como una capa añadida a la superficie (una coloración que no es inherente a su naturaleza material), aportar información adicional sobre el modo en el que funciona el edificio o ha sido ideado, atender únicamente al atractivo visual de la edificación, mimetizarlo con el entorno (confusión del objeto con su contexto), o simplemente cubrirlo de blanco.

\section{Resultados: Casos de estudio}

El primero de los arquitectos a analizar es Bruno Taut (Königsberg, 1880-1938) ${ }^{1}$ que, aunque se relaciona con el movimiento Expresionista, tiene obras de madurez

${ }^{1}$ El arquitecto y publicista soviético se consideró uno de los máximos representantes del Expresionismo alemán, un movimiento en el que se anhelaba alcanzar libertad y mayores niveles de expresión a través del arte y la arquitectura. Entre 1921 y 1924 realizó varios edificios funcionales en Berlín, trabajó como arquitecto municipal en Magdeburgo (Alemania) y en 1932 se trasladó a Moscú. Fue reconocido por su visión utópica y la lucha contra la estética tradicional (Círculo de Bellas Artes, s.f.). 
conectadas con el Movimiento Moderno, a pesar de no ser uno de sus arquitectos más relevantes. Según José Manuel García (2002), Taut destaca por ser un arquitecto implicado con la sociedad, además de por emplear el color sin un aparente patrón o reglas; una metodología que se relaciona con el uso del color de manera lúdica y expresiva, algo propio de los arquitectos modernos, en opinión de David Rivera (2015). Después se revisa uno de los proyectos de Charles-Édouard Jeanneret-Gris, conocido como Le Corbusier² (Suiza, 1887-1965), quien es considerado el padre de la arquitectura moderna, del que se destaca la dualidad en su relación con el color, ya que inicialmente se opone a la aplicación de este en la arquitectura (etapa en la que sobresale por sus arquitecturas blancas); pero al final de su vida, entre los años 30 y 40 , su pensamiento se torna a favor de su uso, viendo un potencial en el color que durante años le había parecido un ataque hacia la arquitectura (Serra, 2013).

El empleo del color en las arquitecturas de la ciudad-jardín Gartenstadt Falkenberg (Figura 2) proyectada por Taut, tiene el objetivo de "dotar de vida a un plan constructivo que, aunque resuelto del modo más adecuadamente científico, no deja de resultar esquemático" (García, 2002, p.9). De manera que este elemento pasa a ser el protagonista, trascendiendo la identidad colectiva, diferenciando las viviendas y provocando de manera consciente un estilo de vida no convencional. El crítico e historiador de arte Adolf Behne, según García (2002), describe la aplicación de color en este conjunto residencial como un hito que rompe la hilera de viviendas recubiertas de blanco.

Taut, uno de los más intrépidos dentro del gremio de los arquitectos por el uso del color, aplicaba este elemento en revocos, muros, puertas y otros elementos de carpintería, con el objetivo de hacer única a cada arquitectura (Serra, 2010). Gracias a esta característica forma de aplicar el color, las viviendas podían individualizarse según su estética, puesto que cada diseño era diferente, llegando incluso a ser un medio para asociar a sus residentes en un contexto de construcciones de tipo social, cuyo diseño arquitectónico formalmente era muy similar (Serra, 2010). El color, según el autor, y como apuntara Van Doesburg en 1983, era una herramienta con la que Taut conseguía dar una estética lúdica y hacer más agradable los bloques de viviendas sociales que se caracterizaban por la repetición modular, la cual aportaba frialdad a la construcción. Azules, rojos, amarillos, verdes, ocres, naranjas (todos ellos con un alto valor cromático) se aplicaban en el revestimiento liso de dichas construcciones. Asimismo, se les añadía pequeñas superficies de color negro que remarcaban algunos elementos como las ventanas, marcos de puertas o tejados (Rivera, 2015) (Figuras 3 y 4).

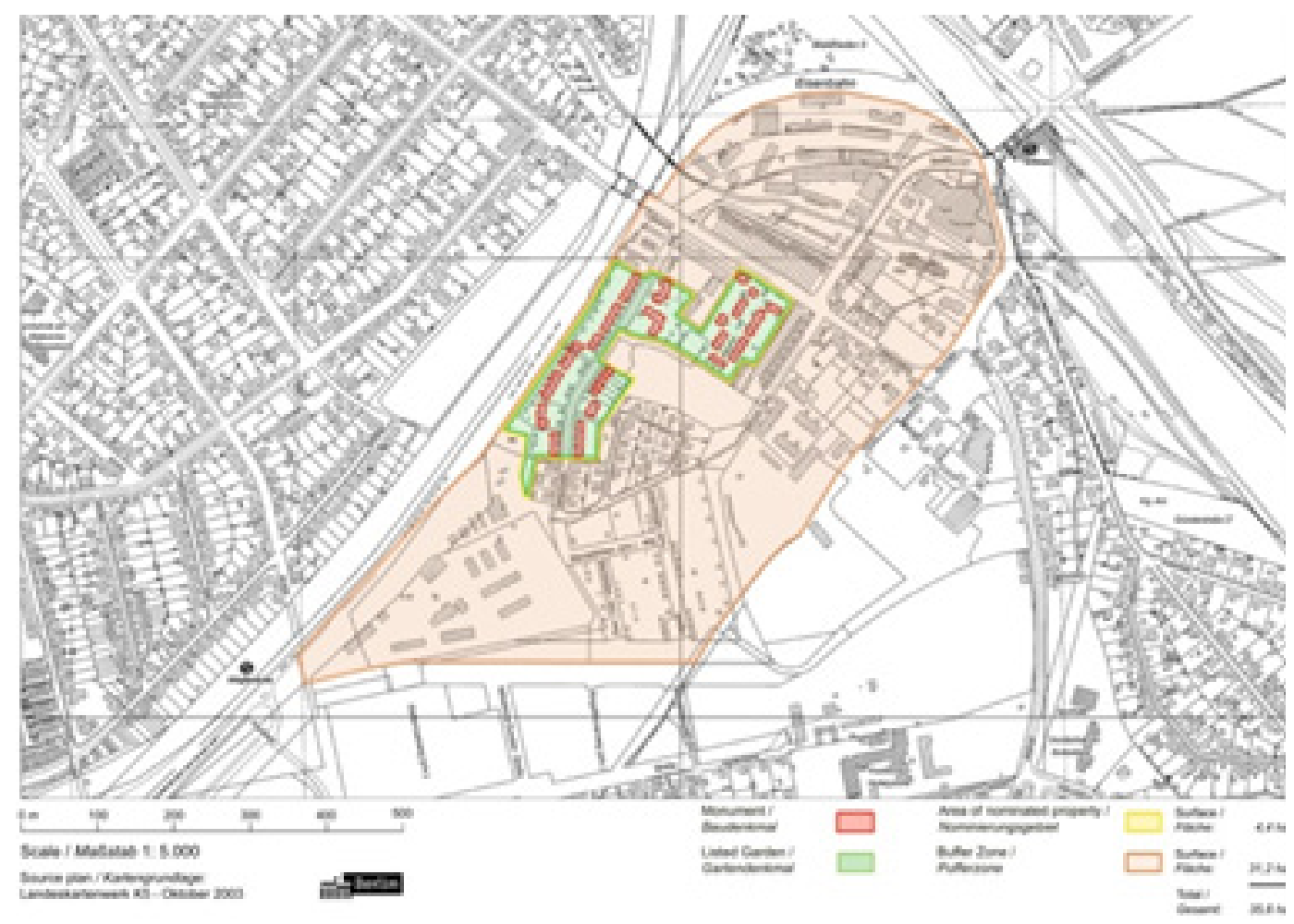

Figura 2 : Bruno Taut, Gartenstadt Falkenberg, 1920-30. Plano cartográfico

Fuente:https://www.berlin.de/landesdenkmalamt/welterbe/welterbestaetten/siedlungen-der-berliner-moderne/ gartenstadt-falkenberg-654609.php

\footnotetext{
2 La tendencia industrial del momento influyó directamente en el estilo de la arquitectura de Le Corbusier, creando obras que él mismo denominaba como "máquinas de habitar" (Obra y biografía de Le Corbusier, 2006)
} 


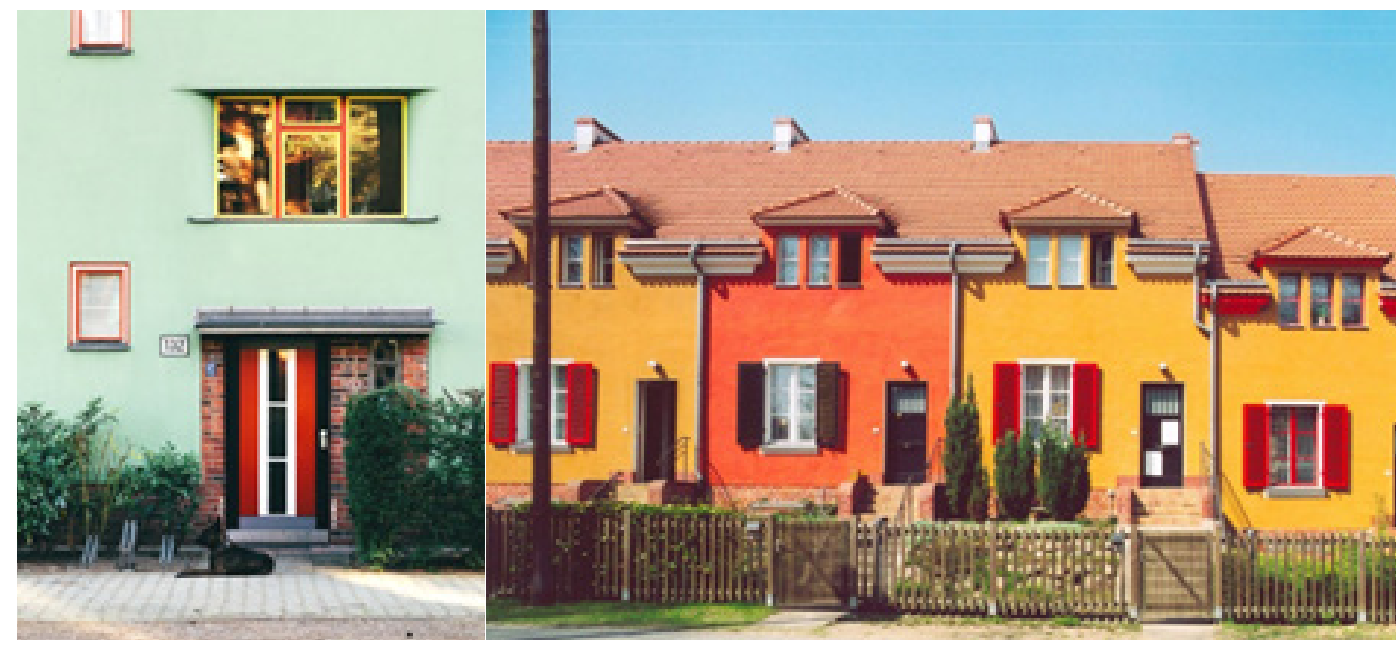

Figuras 3 y 4: Bruno Taut, Gartenstadt Falkenberg, años 20-30. Imágenes del exterior de distintas zonas del barrio. Gartenstadtweg (Alemania)

Fuente: https://www.greyscape.com/siedlungen-der-berliner-moderne/

Pero el color no es perdurable y su estado va cambiando fruto de la erosión del paso del tiempo y los efectos de las condiciones climatológicas, de modo que en 1991 se procedió a la restauración de la coloración (Figuras 5 y 6 ).
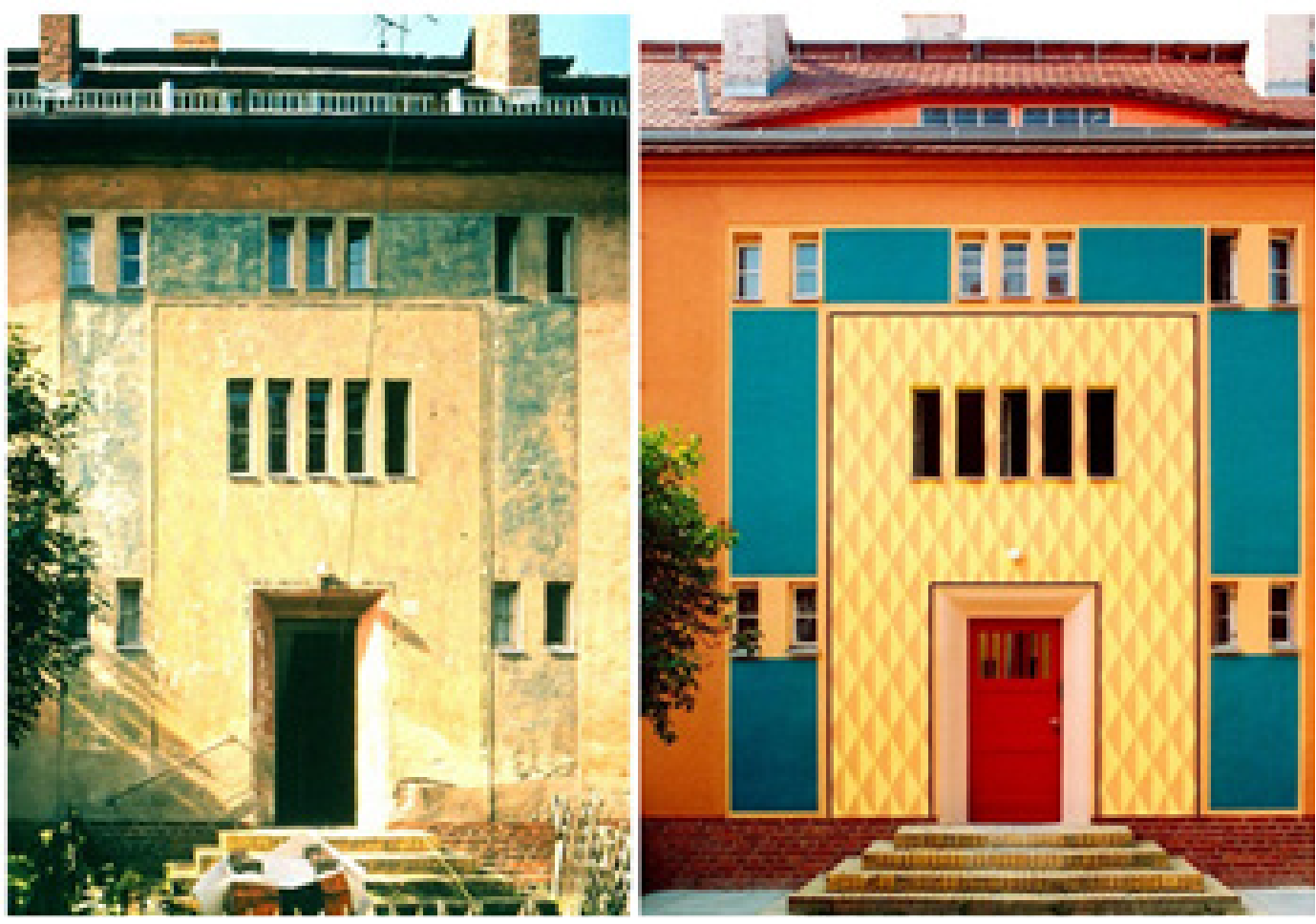

Figuras 5 y 6: Bruno Taut, Gartenstadt Falkenberg, 1920-1930. Imagen de la fachada de una de las viviendas antes y después de la restauración

Fuente: https://www.brenne-architekten.de/gartenstadt-falkenberg/ 
Para Van Doesburg (1985), esta forma de aplicar el color que identificaba el proyecto de Taut Gartenstadt Falkenberg, no seguía ningún patrón ordenado, repetitivo o relacionado con algo; el color, simplemente se distribuía de manera libre y arbitraria, consiguiendo que esas masas de bloques similares fuesen más agradables para la vista.

En relación con este característico diseño de coloración arquitectónica presentado por Taut, se puede decir que en el proyecto se aplica el color por su valor intrínseco, según Juan Serra (2010), con un valor estético notable en relación al resto de viviendas del barrio. En cuanto a los modos de coloración explicados por Malvina Arrarte (2008), se relaciona con la aplicación del color de forma plana, capa y decorativa, puesto que el color consigue reducir al objeto arquitectónico a los elementos planos de los que se compone. La pintura se aplica sobre la superficie, potenciando el aspecto estético de la arquitectura, adquiriendo en este caso concreto (por el uso de elementos geométricos repetitivos) un aspecto fragmentado de la pared, que a su vez caracteriza o define un estilo propio para cada conjunto de edificaciones, reconocible dentro del barrio.

Pero no todos estaban de acuerdo con esta estética tan colorida con la que se había presentado la arquitectura del barrio de Gartenstadtweg. Según Alberto Pizza (1994), se decía entre la sociedad alemana que la exacerbada utilización del color y la combinación de tonos en las creaciones de Taut, provocaban en los berlineses un estado semejante al de rebelión. Por esta razón el arquitecto fue sentenciado por la propia sociedad, catalogándolo como loco, llegando incluso a pedir su encarcelamiento. En su defensa Taut, según Juan Serra (2010), alegó que coloreaba la arquitectura porque el gris del recubrimiento de las construcciones del siglo XIX envolvía a los habitantes en un ambiente de tristeza y no fomentaban la productividad y el goce de la vida. Coincidimos con Taut en esta percepción de la arquitectura gris, puesto que su neutralidad no atrae la atención de la ciudadanía ni la estimula sensorialmente. Por eso se requiere del color para activar, tanto la arquitectura como a su entorno, implicando también al público.

Continuamos con el análisis de diseños cromáticos llevados a cabo dentro del Movimiento moderno, centrándonos en la producción de Le Corbusier. Este arquitecto, en su debate por conseguir una fórmula adecuada para aplicar color a la superficie arquitectónica o, mejor dicho, para colorear la arquitectura, define el color junto a su compañero, el profesor de arquitectura suizo Arthur Rüegg, como un elemento que induce en el espectador reacciones psicológicas profundas e influye en sus sentimientos, estando íntimamente unido al ser (Rüegg y Le Corbusier, 1997). Para estos arquitectos no todos los tonos ejercen la misma acción sobre idéntico sentimiento. Por ejemplo, al azul lo asocian con la sensación subjetiva de suavidad y calma, mientras que el rojo actuaría como un estimulante (descanso vs acción). En cuanto a la expresión del volumen, conciben el color como un agente peligroso que puede destruirlo o desorganizarlo (Rüegg y Le Corbusier, 1997). La solución a esta situación fue propuesta por el propio Le Corbusier, en colaboración con el pintor Amédée Ozenfant.
Consistió en ordenar, clasificar y establecer una jerarquía cromática en el diseño arquitectónico, una distribución a la que denominaron "escala dinámica", formada por tres subescalas: mayor, dinámica y de transición, según apunta David Batchelor (2000). Este autor señala igualmente que Le Corbusier y Ozefant abordaron el tema del color en la arquitectura en diversos números de la revista francesa L'Esprit Nouveau (1921, 1923 y 1924), en los que desarrollaron teóricamente la relación directa entre color y forma, contemplando la posibilidad de poder aplicar el primero a posteriori de la creación de la segunda.

La escala mayor se establecía con los tonos que para Ozenfant y Le Corbusier eran constructivos, es decir, que transmitían fortaleza, estabilidad, unidad y equilibrio, como los amarillos ocres, rojos, tierras, blanco, negro, azul ultramarino y derivados de estos. A la escala dinámica pertenecían los tonos amarillo limón, naranjas, bermellón y otros colores muy luminosos, los cuales, según Le Corbusier, estorbaban en la construcción porque eran demasiado potentes para la vista, llegando a ser incluso cegadores; estos tonos, por tanto, debían emplearse en menor medida que los pertenecientes a la escala mayor. En el caso de la escala de transición, esta contenía colores que no se consideraban aptos para la construcción, como, por ejemplo, el verde esmeralda, porque no se encontraban con frecuencia en la naturaleza. Esta escala es la que se empleaba en menor proporción respecto a las dos anteriores (Batchelor, 2000).

En el caso de los Quartiers Modernes Frugès (1924-1926) (Figuras 7-14), predominan los tonos naranja (pardosrojizos), verde esmeralda claro (aguamarina), blanco y azul claro, pertenecientes a las tres escalas, siendo de la primera (o escala mayor) el color blanco y el azul claro (colores constructivos); el naranja pertenece a la escala dinámica y el verde esmeralda claro a la escala de transición. En esta etapa de la trayectoria profesional del arquitecto comienza a probar el color en grandes planos, formando parte del revestimiento exterior de las viviendas. Estos planos, tal y como se puede ver en las imágenes, se adaptan a las formas de la pared y quedan limitados por sus dimensiones, sin extenderse a paredes contiguas.

Situada al suroeste de Francia, en Pessac, suburbio de la ciudad de Burdeos, este barrio residencial diseñado para albergar a los obreros de la fábrica azucarera del poderoso industrial Henri Frugès, destacaba por el emplazamiento rodeado de bosque además de por la situación cercana a las industrias, las vías del tren y el hospital de tuberculosos (una de las enfermedades más frecuentes de la época) (Ragot, 2019). El objetivo era que todos los obreros tuvieran una vivienda digna, construida a partir de varios modelos normalizados en hormigón armado, construidos en serie mediante procesos industriales (Figuras 7 y 8). Las indicaciones del propio Frugès fueron bastante claras en este sentido:

(...) les pido que se planteen el problema de la planificación de la vivienda, que encuentren la estandarización adecuada, usando muros, suelos, techo, de acuerdo con la más rigurosa solidez y eficacia, prestándose a una verdadera taylorización mediante el empleo de máquinas que les autorizo a adquirir. (Citado en Boesiger, 1982, p. 28) 


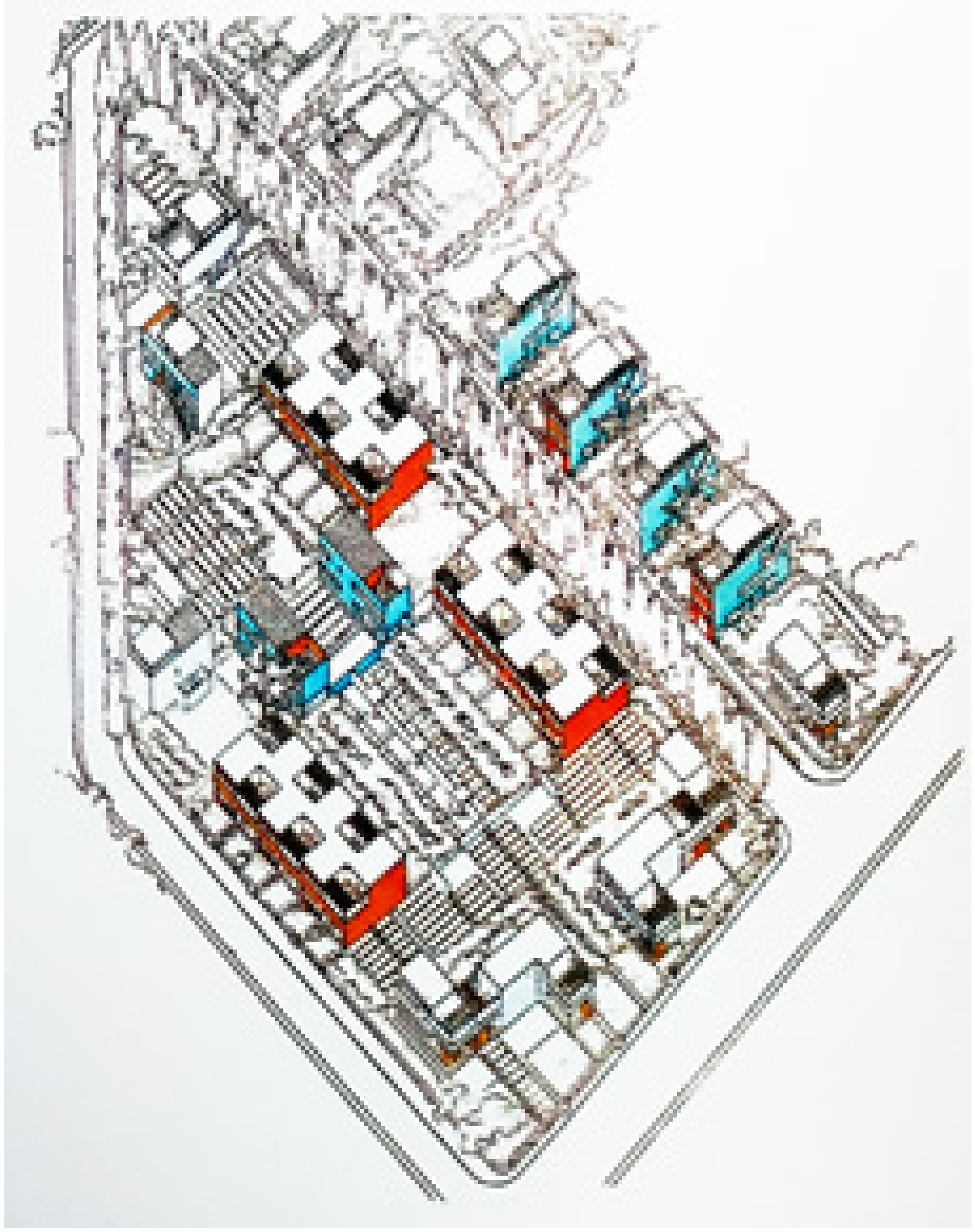

Figura 7: Le Corbusier, Quartiers Modernes Frugès, 1924-1926. Detalle de la planta en axonométrica. Fuente: Cohen, J. L. (2004). Le Corbusier. Taschen.

A pesar de que la intención inicial era conseguir un modelo de vivienda económico que permitiese su reproducción a gran escala, democratizando así el acceso a la misma ${ }^{3}$, la dificultad técnica de su construcción debido a su carácter innovador (utilización de hormigón proyectado, difícil de manejar, de ventanas modulares que no siempre

${ }^{3}$ Wiliam J. R. Curtis indica, en este sentido, que Le Corbusier confiaba en "las virtudes sociales de las viviendas económicas" (1987, p. 28), teniendo dos oportunidades para construirlas, ambas en Burdeos, $y$ contando con Henri Frugés como promotor: las construidas en Pessac (1924-1926) y en Lège-Cap Ferret (1923), ambas amparadas por la Ley Ribot de 1908. encajaban en los vanos habilitados, coste elevado de la maquinaria inicial necesaria...), provocó el encarecimiento de las residencias que integraban el complejo, siendo prohibitivo para los trabajadores de Frugès (Curtis, 1987). Este hecho, sumado a problemas con la licencia de obras, llevaron al promotor y empresario de Burdeos a la quiebra y, por tanto, solo pudieron construirse 53 de las 135 viviendas proyectadas. Además, los bombardeos de la Segunda Guerra Mundial destruyeron tres de estas viviendas, con lo cual el proyecto se quedó en apenas 50 viviendas (Ragot, 2019). 


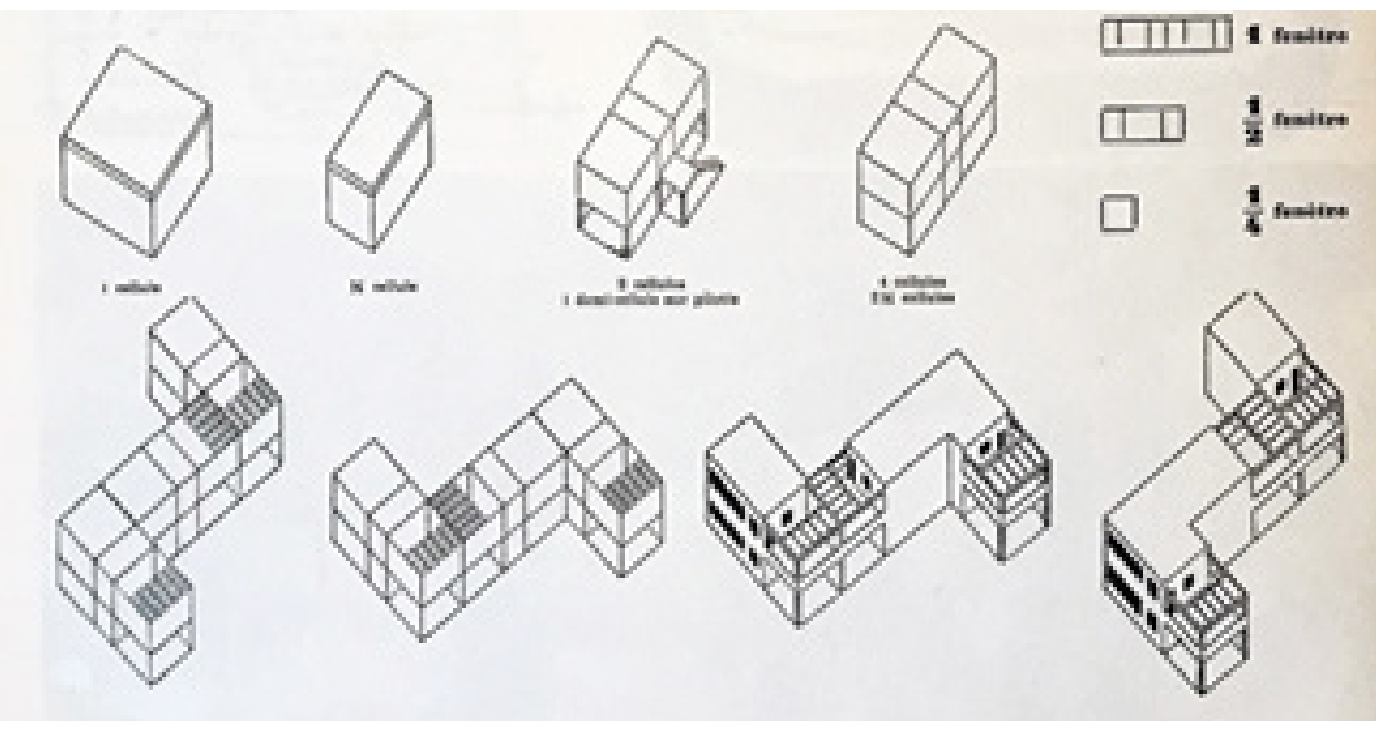

Figura 8 : Le Corbusier, Quartiers Modernes Frugès, 1924-1926. Esquema del planteamiento de vivienda estandarizada a partir del cubo

Fuente: Boesiger, W. (1982). Le Corbusier (5a ed.). Gustavo Gili

Le Corbusier propuso varios diseños para esta ciudad jardín, los cuales llevaba estudiando desde 1914 con su primo Pierre Jeanneret (Suiza, 1896-1967). Todos ellos partían del cubo como célula base que era dividida o multiplicada en función de las necesidades y de las plantas finales. Las ventanas también respondían a una estructura modular cuadrangular con la que se jugaba, creando diferentes ritmos en las fachadas (Figura 8) Las arquitecturas se clasificaron según su estilo formal en: Zig-Zag (Figura 9), Quinconces (Figura 10), Jumelle (Figura 11), Gratte-ciel (Figura 12), Arcade (Figura 13) e Isolée $e^{4}$ (Figura 14). Estos estilos tienen diferentes diseños cromáticos agrupados entre sí. En las casas estilo Zig-Zag y Quinconces se aplican los tonos azul claro, naranja y blanco, mientras que en las casas Jumelle, Gratte-Ciel e Isolée se incorpora el tono verde esmeralda claro a los antes mencionados y se suprime el azul claro. En el caso de las casas estilo Arcade solo se aplican los tonos blanco, naranja y verde esmeralda claro, que además forma parte de las ventanas.

La selección de estos tonos lleva a pensar en la aplicación de unas estrategias relacionadas con la descripción formal del objeto apuntada por Serra (2010), puesto que el color interfiere en la percepción del volumen del edificio al extenderse cada tono únicamente por el plano de una pared, evitando su repetición en paredes contiguas. Este hecho provoca que dos colores se encuentren en la esquina, evocando cierta sensación de inmaterialidad, como sugiere el arquitecto danés Steen Eiler Rasmussen, cuando visita el conjunto residencial en 1926 (Curtis, 1987). En cuanto a los modos de coloración, según Malvina Arrarte (2008) se puede decir que se aplican los de tipo plano, capa y decorativo, porque el color reduce el objeto a los elementos planos de los que se compone; la capa añadida anula el material de construcción, pero a mismo tiempo potencia su estética. Asimismo, el diseño

4 Zig-zag, Damero, Gemela, Rascacielos, Arcadas y Aislada (traducción según las autoras) cromático planteado ayuda a identificar los diferentes estilos presentes en esta ciudad jardín.

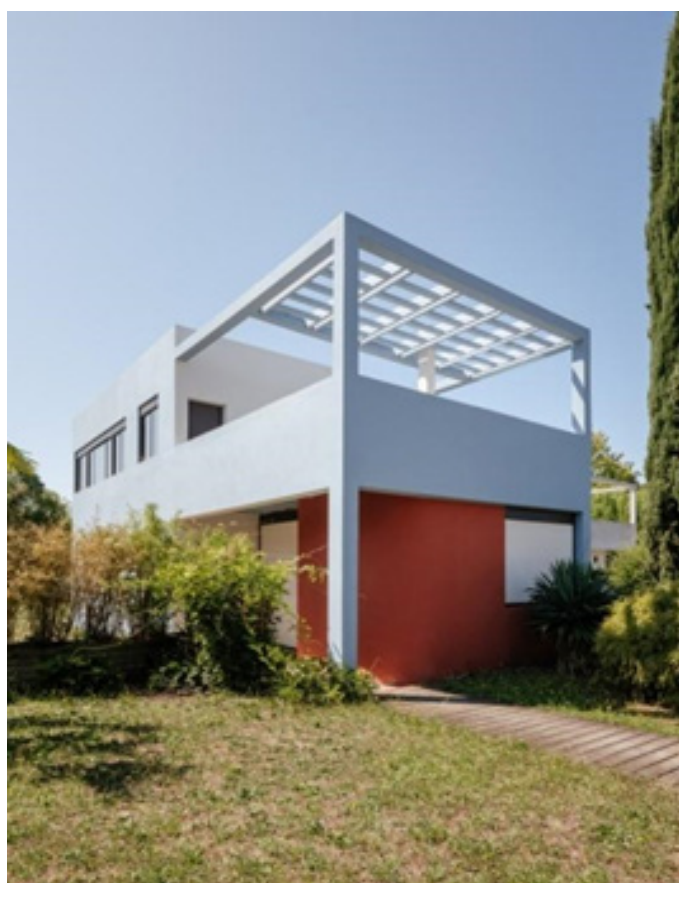

Figura 9 : Le Corbusier, Maison Zig-Zag, 1926. Quartiers Modernes Frugès. Pessac (Francia)

Fuente: https://www.plataformaarquitectura.cl/ cl/940544/cite-fruges-de-le-corbusier-leccionesde-un-barrio-de-viviendas-sociales-modernas?ad medium=gallery 


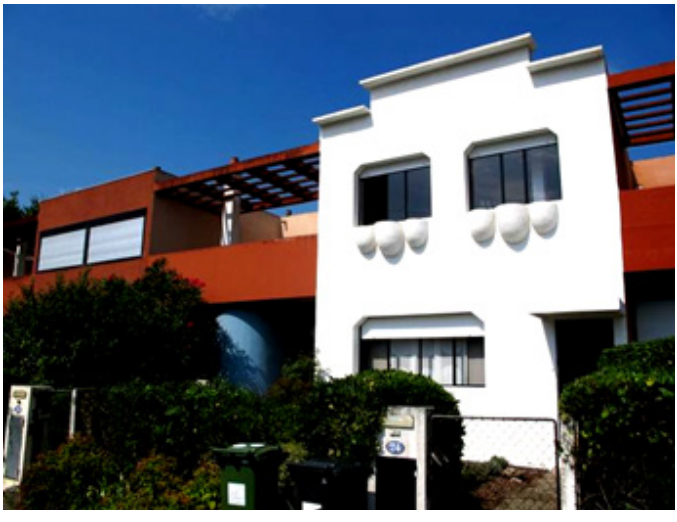

Figura 10: Le Corbusier, Maison Quinconces, 1926. Les Quartiers Modernes Frugès. Pessac (Francia)

Fuente: https://www.serendipidoc.fr/bibliotourisme-lecorbusier-en-gironde/corbu9/

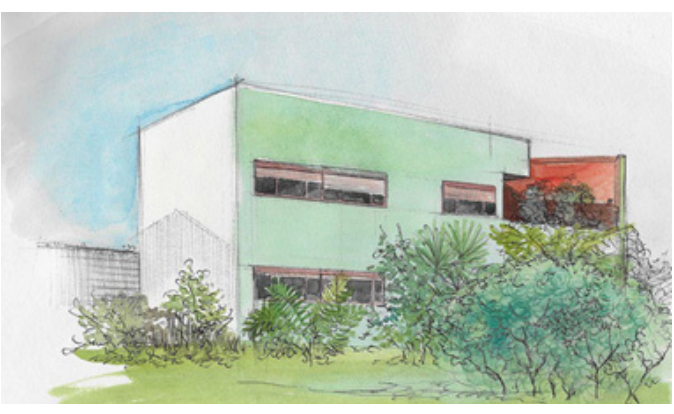

Figura 11: Le Corbusier, Maison Jumelle, 1926. Acuarela de la vista exterior y fotografía de una de las habitaciones. Les Quartiers Modernes Frugès. Pessac (Francia)

Fuente: http://la-galerie-d-helene.over-blog. com/2016/12/maison-jumelle-cite-fruges-de-lecorbusier.htm

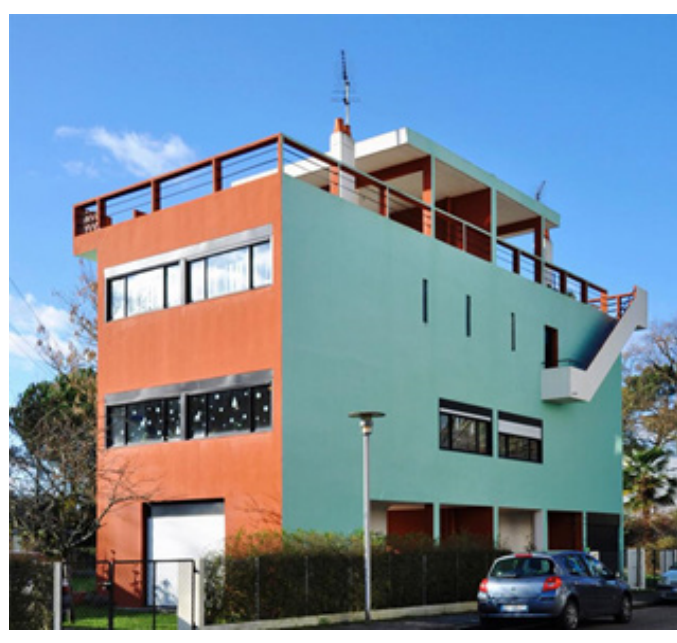

Figura 12: Le Corbusier, Maison Gratte-ciel, 1926. Les Quartiers Modernes Frugès. Pessac (Francia)

Fuente: https://architecturalvisits.com/la-cite-frugesles-quartiers-modernes-fruges/

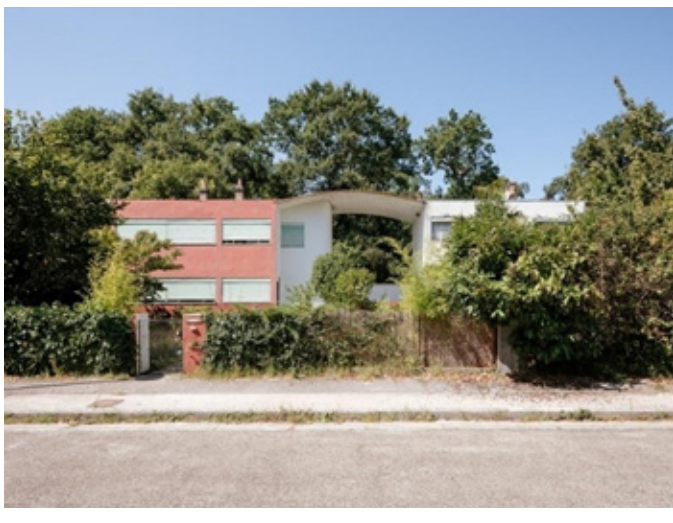

Figura 13 : Le Corbusier, Maison Arcade, 1926. Les Quartiers Modernes Frugès. Pessac (Francia)

Fuente: https://architecturalvisits.com/la-cite-fruges les-quartiers-modernes-fruges/

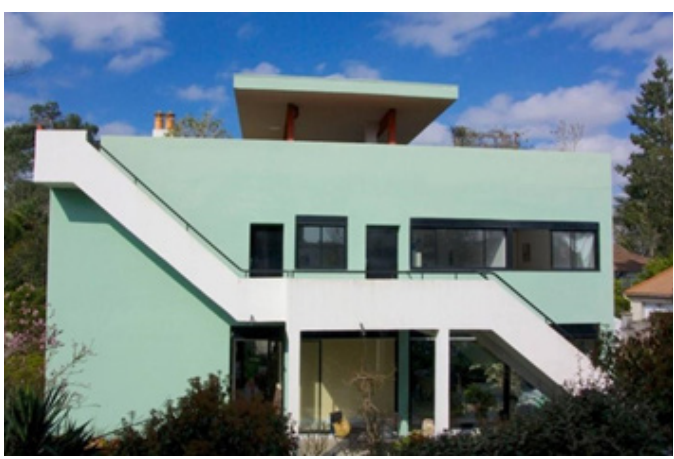

Figura 14 : Le Corbusier, Maison Isolée, 1926. Vista de la fachada trasera de la vivienda. Les Quartiers Modernes Frugès. Pessac (Francia)

Fuente: http://architectuul.com/architecture/cite-fruges

\section{Conclusiones}

En los casos de estudio revisados la arquitectura es coetánea al momento en el que se aplica la coloración, aunque en ocasiones esta ya esté construida y el diseño se haya planteado posteriormente. Es decir, el color se aplica a una base que tiene un estilo perteneciente al Movimiento moderno, donde se rompe con el estilo clásico arquitectónico, predominantemente ornamental, y se potencian las formas y volúmenes geométricos y rectilíneos gracias a materiales como el hierro, la madera, el vidrio y el hormigón; por eso el revestimiento exterior de la construcción no tiene ningún elemento ornamental en relieve. La ornamentación se puede simular mediante la forma y el color, como ocurre en el ejemplo de Gartenstadt Falkenberg, donde el arquitecto Bruno Taut crea un diseño cromático con aspecto decorativo $y$, a la vez, diferenciador, porque cada construcción tiene asociado uno distinto. Estas aportaciones cromáticas activan cada una de las viviendas y les otorgan personalidad, influyendo igualmente sobre el estado anímico de los obreros que residen en ellas. 
Al comparar cómo Bruno Taut y Le Corbusier proyectan la aplicación del color en cada uno de los conjuntos residenciales analizados, se observa que la forma de proceder difiere bastante entre ellos; mientras que el primero opta en algunos casos por motivos o patrones mediante los que fragmenta la pared en multiplicidad de formas geométricas y colores, el segundo se decanta por la articulación de planos de color que se ajustan a las dimensiones de la pared. La mínima presencia del color blanco en las casas de Taut, frente a su inclusión en todas las de Le Corbusier, también es un punto de contraste entre ambos creadores. No obstante, uno y otro coinciden en disponer el color de manera plana y homogénea, evitando la generación de cualquier textura visual o táctil, que en Le Corbusier tiene reminiscencias de la pintura purista del momento (Cohen, 2004; Curtis, 1987).

Por otra parte, en cuanto a la distribución de los planos de color y la utilización de una determinada gama cromática, se plantea una cuestión que afecta a la composición arquitectónica: el uso de la monocromía como una estrategia. En el caso de la coloración arquitectónica aplicada de manera uniforme, monocroma y siguiendo el plano rectangular de cada paño del edificio, como se aprecia en el proyecto Quartiers Modernes Frugès y en algunas viviendas de la urbanización Gartenstadt Falkenberg, lleva a la desmaterialización de la arquitectura, eliminando visualmente todos los elementos ornamentales y funcionales (puertas, ventanas, rejas, etc.) que pueda contener el recubrimiento exterior de la construcción; por tanto, con esta técnica de coloración se potencia el color como elemento independiente de la arquitectura sobre la que se aplica. Por otra parte, la sensación que producen algunas de las fachadas de las casas que forman parte del Gartenstadt Falkenberg es que son abordadas cromáticamente como si fueran un lienzo, articulando la composición, teniendo en cuenta los diferentes elementos que las integran, como puertas, ventanas, marcos, molduras etc., incluyéndolos cromática y formalmente en el diseño final. Los dos ejemplos analizados constituyen, por tanto, diversas maneras de abordar el cromatismo en la arquitectura moderna, proponiendo soluciones diferentes para un mismo fenómeno.

Cómo citar este artículo/How to cite this article: Alcaide-Ramírez, A. y Ruiz-Abellón, A.(2021). La aplicación del color sobre la arquitectura moderna: Revisión de los proyectos de Bruno Taut y Le Corbusier. Estoa. Revista de la Facultad de Arquitectura y Urbanismo de la Universidad de Cuenca, 10(20), 153-164. doi: 10.18537/est.v010.n020.a13

\section{Referencias bibliográficas}

Arrarte, M. (2008). On the Bonding of Colour $y$ Architecture: Colouring Modes. International Colour Association.

Batchelor, D. (2000). Cromophobia. Reaktion Books Ltd.

Boesiger, W. (1982). Le Corbusier (5a ed.). Gustavo Gili.

Círculo de Bellas Artes (s.f.). Bruno Taut. https://www. circulobellasartes.com/biografia/bruno-taut/

Cohen, J. L. (2004). Le Corbusier. Taschen.

Curtis, W. J. R. (1987). Le Corbusier. Ideas y formas. Hermann Blume.

Diecisiete puntos de la arquitectura neoplasticista (2012, 19 de enero). Tecnne. https://tecnne.com/de-stijl/17puntos-de-la-arquitectura-neoplasticista/

García, J.M. (2002). Arquitectos alemanes, arquitextos desconocidos. Bruno Taut (1880-1938). Cuadernos de Instituto Juan de Herrera de la Escuela de Arquitectura de Madrid

Hernández, R., Fernández, C. y Baptista, P. (2010). Fundamentos de metodología de la investigación. McGraw-Hil

Mehaffy, M. y Salingaros, N. (2020, 23 de febrero). La arquitectura moderna imperialista ataca de nuevo (J. T. Franco, Trad.). Plataforma arquitectura. https://www. plataformaarquitectura.cl/cl/933905/la-arquitecturamodernaimperialista-ataca-de-nuevo

Mondrian, P. (1983). La nueva imagen en la pintura. La realización del neoplasticismo en la arquitectura del futuro lejano y de hoy. Colegio Oficial de Aparejadores y Arquitectos Técnicos de Murcia

Mújica, V. (2014, 1 de febrero). Vanguardias III. Historia de la arquitectura moderna. http:// historiadearquitecturamoderna.blogspot.com/2014/02/ vanguardiasiii.html

Munárriz, J. (2013). Investigación y tesis doctoral en Bellas Artes. E-Prints Complutense.https://eprints.ucm. es/23022/1/Investigaci\%C3\%B3nTesisBBAA_Jaime_ Mun\%C3\%A1rriz.pdf

Pizza A. (1994). Después del cubismo. El Croquis, (4), 8-47

Premier, A. y Gasparini, K. (2017). Sustainable colour design in architecture: materials, technologies and products. Journal of the International Colour Association, 19, 34-46.

Ragot, G. (2019). Le Corbusier's paradoxical appropiation in Pessac, France. En M. Corbin, I. Gournay y R. Freestone (Eds.), Iconic planned communities and thechallenge of change (pp. 238-322). University of Pennsylvania Press. 
Rivera, D. (2015). Los poderes del leopardo. Colores, moral y arquitectura de John Ruskin a Frank Gehry. En D. Rivera, Color, textura y arquitectura contemporánea (pp.11-22). Baumit. http://oa.upm.es/49395/1/Rivera Poderes_leopardo.pdf

Rüegg, A. y Le Corbusier (1997). Le Corbusier's Color Keyboardsfrom 1931 and 1959. Birkhäuser

Sainz, J. (1997). Arquitectura y urbanismo del siglo XX. En J. Brihuega, J. Hernando Carrasco, J.A. Ramírez, T. Raquejo Grado, C. Reyero, J. Sainz, F. J. San Martín, y G. Solana (Eds.), Historia del arte 4. El mundo contemporáneo (pp. 265-335). Alianza Editorial. https://doi.org/10.13140/ RG.2.1.1235.2248

Serra, J. (2013). Three color strategies in architectura composition. Color Research and Application, 38 (4), 238 250. http://dx.doi.org/10.1002/col.21717

Serra, J. (2010). La versatilidad del color en la composición de la arquitectura contemporánea europea: contexto artístico, estrategias plásticas e intenciones [Tesis Doctoral, Universidad Politécnica de Valencia]. TESEO. https:// www.educacion.gob.es/teseo/imprimirFicheroTesis. do?idFichero=\%2FappnNbNLRQ\%3D

Torrico, E. (2020, 29 de junio). ¿Casas y escuelas anticovid? Cómo la arquitectura pospandemia ha moldeado siempre nuestras ciudades. El Confidencial. https://www.elconfidencial.com/multimedia/video/ cultura/2020-06-29/tuberculosis-y-covid-escuelas-airelibre-casas-terrazaspandemia_2658575/

Tournikiotis, P. (2001). La historiografía de la arquitectura moderna. En J. Sainz (Ed. y Trad.). Manuales universitarios de arquitectura 5. Celeste Ediciones SA.

Van Doesburg, T. (1985). La evolución de la Arquitectura Moderna en Holanda. En T. Van Doesburg (Ed.) Principios del nuevo arte plástico y otros escritos, (pp. 111-119). Colegio Oficial de Aparejadores y Arquitectos Técnicos de Murcia.

Villalobos, D. (2002). El color de Luis Barragán. Morés. 